\title{
Genetic Associations Analysis in Tomato (Solanum lycopersicum L.) Involving Improved Germplasm Lines for Agronomic and Yield Contributing Traits
}

\author{
M.K. Sunilkumar ${ }^{1}$, S. Vijeth ${ }^{2}$, Vijayakumar Rathod ${ }^{1}$ and Prashant Kaushik ${ }^{3 *}$
}

${ }^{1}$ Division of Vegetable Science, University of Horticultural Sciences, Bagalkot 591 310, India

${ }^{2}$ Department of Vegetable Science, Kerala Agricultural University, Vellayani 695 522, India

${ }^{3}$ Instituto de Conservación y Mejora de la Agrodiversidad Valenciana, Universitat

Politècnica, de València, Valencia 46022, Spain

*Corresponding author

\section{A B S T R A C T}

\section{Keywords}

Correlation and path analysis, Growth, Earliness, Quality and yield traits, Tomato

Article Info

Accepted:

25 September 2019

Available Online:

10 October 2019
Correlation and path analysis was carried out in 60 tomato genotypes using growth, earliness, quality and yield characters. Very high (>40\%) genotypic coefficient of variation (GCV) and phenotypic coefficient variation (PCV) were observed for fruit volume, average fruit weight and yield plant-1. It indicates existence of broad genetic base, which would be amenable for further selection. Very high heritability $(>90 \%)$ coupled with very high genetic advance as per cent over mean (>40\%) was recorded for the characters viz., polar diameter, fruit volume, average fruit weight, number of fruits plant-1, yield plot1.Yield per plant was positively and significantly associated with average fruit weight, fruit volume, equatorial diameter, pericarp thickness, polar diameter and number of locules. Yield per plant was negatively and significantly associated with number of branches at 90 DAT, number of branches at 60 DAT, plant height at 90 DAT and plant spread from north to south at 60 DAT. Path analysis revealed that number of fruits per plant followed by plant spread from north to south at 60 DAT, plant spread from east to west at 60 DAT, average fruit weight and fruit volume. Hence, direct selection for these traits is suggested for yield improvement.

\section{Introduction}

Tomato is an important member of family Solanaceae. For a systematic breeding program, it is essential to identify the parents as well as crosses to bring the genetic improvement in economic character (Kaushik and Dhaliwal, 2018). The magnitude of heterosis depends on the genetic diversity existing between the parents. In a crop like a tomato, where there are evidences for polygenic action determining the yield, and the yield components the choice of parents must be based on refined biometrical techniques (Vijeth et al., 2019).

The value of genotypes depends on the ability to produce superior hybrids in combination with other genotypes (Kaushik, 2015). In tomato to exploit the available variability 
through the breeding program, the genetic study regarding the yield and quality trait is essential.

The yield in tomato is due to the interaction between many of the correlated characters. Selection of these characters is very important when based on the component characters which will be highly heritable and also positively correlated (Kaushik et al., 2015). The correlation coefficient method of analysis helps to identify the mutual relationship between several characters and it also helps to identify the component traits on which selection can be relied. Correlation studies provides information on all characters which are associated with yield.

Ahybrid possessing higher yield, better quality will be an important contribution to farmers. An ideal chilli hybrid should be vigorous, have good branching habit, early flowering, prolonged production of flowers, high fruit weight, good plant height and high yield potential (Kaushik, 2019a and Kaushik, 2019b). It may be difficult to develop a hybrid having all these characters, but it is reasonable to develop one which can have maximum number of desirable characters keeping yield as a primary motto.

\section{Materials and Methods}

Sixty genotypes collected from different sources were evaluated during 2014-15 in the Department of Vegetable Science, Kittur Rani Channamma, College of Horticulture, Arabhavi. Arabhavi is situated in Northern dry zone of Karnataka State at 16o 12' North latitude, 74o 54' East longitude and an altitude of 640 meters above the mean sea level. Arabhavi, which comes under the Zone3 of Region- 2 among the agro-climatic zones of Karnataka, has benefits of both the southwest and north-east monsoons. Genotypes used in this experiment with their sources of collection are listed in Table 1. The crop was grown in a randomized block design with two replications at spacing of $90 \times 60 \mathrm{~cm}$. Five randomly chosen plants in each replication of each genotype were labelled and used for recording the observations. Genotypic correlation coefficients were worked out among different traits using per se values $(n=120)$. Correlations and path analysis carried out according to procedure given by Dewey and Lu (1959) respectively.

\section{Estimation of genetic variability parameters}

\section{Genotypic and phenotypic coefficient of variation}

Genotypic and phenotypic coefficients of variance were estimated according to Burton and Devane (1953) based on estimate of genotypic and phenotypic variance.

\section{Genotypic co-efficient of variation (GCV)}

$\operatorname{GCV}(\%)=\frac{\sigma g}{\mathrm{X}}$

Phenotypic co-efficient of variation (PCV)

$$
\operatorname{PCV}(\%)=\frac{\sigma p}{X}
$$

Where,

$\mathrm{X}=$ General mean

$\sigma g=$ Genotypic standard deviation

$\sigma \mathrm{p}=$ Phenotypic standard deviation

GCV and PCV were classified as suggested by Burton and Devane (1953)

$\begin{array}{ll}0-10 \% & : \text { Low } \\ 10-20 \% & : \text { Moderate } \\ 20 \% \text { and above: } & \text { High }\end{array}$




\section{Heritability $\left(\mathbf{h}^{2}\right)$}

The broad sense heritability $\left(\mathrm{h}^{2} \mathrm{bs}\right)$ was estimated by following the procedure suggested by Webber and Moorthy (1952) as indicated here below.

$h^{2}=\frac{\sigma^{2} g}{\sigma^{2} p}$

Where, $\mathrm{h}^{2}(\%)=$ Heritability (Broad sense)

$\sigma^{2} \mathrm{~g}=$ Genotypic variance

$\sigma^{2} \mathrm{p}=$ Phenotypic variance

\section{Expected genetic advance}

Genetic advance for each character was predicted by the formula given by Johnson et al., (1955).

$\mathrm{GA}=\mathrm{h}^{2} \times \sigma_{\mathrm{p}} \times \mathrm{k}$

Where, $\mathrm{k}=$ selection differential (2.06) at 5 per cent selection intensity

$\mathrm{h}^{2}=$ Heritability in broad sense

$\sigma_{\mathrm{p}}=$ Phenotypic standard deviation

\section{Genetic advance over per cent of mean (GAM)}

Genetic advance as percentage over mean was worked out as suggested by Johnson et al., (1955).

Genetic advance over mean (GAM) = $\frac{\mathrm{GA}}{\overline{\mathrm{X}}} \times 100$

Where, $\mathrm{GA}=$ Genetic advance $\mathrm{X}=$ General mean

The genetic advance as per cent of mean was categorized as suggested by Johnson et al., (1955) and the same is given below.
0-10\% : Low

11-20\% : Moderate

$21 \%$ and above: High

\section{Correlation analysis}

The correlation co-efficient among all possible character combinations at phenotypic (rp) and genotypic (rg) level were estimated employing formula (Al-Jibouriet al., 1958).

Phenotypic correlation $=r_{x y}(p)=\frac{\text { Covxy }}{\sqrt{V x(p) x V_{y}(p)}}$

Genotypic correlation $=r_{x y}(g)=\frac{\operatorname{Covxy}(\mathrm{g})}{\sqrt{V_{x}(\mathrm{~g}) \times V_{y}(\mathrm{E})}}$

Where,

$\operatorname{Cov}_{\mathrm{xy}}(\mathrm{G})=$ Genotypic covariance between $\mathrm{x}$ and $y$

$\operatorname{Cov}_{\mathrm{xy}}(\mathrm{P})=$ Phenotypic covariance between $\mathrm{x}$ and $\mathrm{y}$

$V_{x}(G)=$ Genotypic variance of character ' $x$ '

$V_{x}(P)=$ Phenotypic variance of character ' $x$ '

$V_{y}(G)=$ Genotypic variance of character ' $y$ '

$V_{y}(P)=$ Phenotypic variance of character ' $y$ '

The test of significance for association between characters was done by comparing table ' $r$ ' values at $n-2$ error degrees of freedom for phenotypic and genotypic correlations with estimated values, respectively.

\section{Path co-efficient analysis}

Path co-efficient analysis suggested by Wright (1921) and Dewey and Lu (1959) was carried out to know the direct and indirect effect of the morphological traits on plant yield. The following set of simultaneous equations were formed and solved for estimating various direct and indirect effects.

$\mathrm{r}_{1 \mathrm{y}}=\mathrm{a}+\mathrm{r}_{12} \mathrm{~b}+\mathrm{r}_{13} \mathrm{c}+\ldots \ldots \ldots \ldots . .+\mathrm{r}_{1 \mathrm{i}}$

$r_{2 y}=a+r_{21} a+b+r_{23} c+\ldots \ldots \ldots+r_{2 l i}$ 
$r_{3 y}=r_{31} a+r_{32} b+c+\ldots \ldots \ldots \ldots \ldots \ldots .+r_{3 l i}$

$\mathrm{r}_{1 \mathrm{y}}=\mathrm{r}_{11} \mathrm{a}+\mathrm{r}_{12} \mathrm{~b}+\mathrm{r}_{13} \mathrm{c}+\ldots \ldots \ldots \ldots \ldots . .+\mathrm{I}$

Where,

$\mathrm{r}_{1 \mathrm{y}}$ to $1_{1 \mathrm{y}}=$ Co-efficient of correlation between causal factors 1 to I with dependent characters y.

$\mathrm{r}_{12}$ to $\mathrm{r}_{11}=$ Co-efficient of correlation among causal factors

$\mathrm{a}, \mathrm{b}, \mathrm{c} \ldots . . \mathrm{i}=$ Direct effects of characters ' $\mathrm{a}$ ' to 'I' on the dependent character ' $y$ '

Residual effect (R) was computed as follows.

Residual effect $(\mathrm{R})=1-\sqrt{\mathrm{a}^{2}+\mathrm{b}^{2}+\mathrm{c}^{2}+}$ ........i $\mathrm{i}^{2}+2 \mathrm{abr}_{12}+2 \mathrm{acr}_{13}+\ldots$.

\section{Results and Discussion}

Very high (> 40\%) genotypic coefficient of variation $(\mathrm{GCV})$ and phenotypic coefficient variation (PCV) were observed for fruit volume, average fruit weight and yield per plant. It indicates existence of broad genetic base, which would be amenable for further selection.

Fruit yield per plant exhibited high positive significant correlation with polar diameter, equatorial diameter, pericarp thickness, number of locules, average fruit weight and fruit volume at both genotypic and phenotypic level. The positive association of these suggests that selection of these traits would result in increased yield. Whereas, Fruit yield per plant exhibited high negative significant correlation with plant height at 60 and 90 DAT, number of branches at 60 and 90 DAT and plant spread from north to south at 60 and 90 DAT. Increased vegetative growth increases the number of fruits per plant but reduced individual fruit size because of increased competition among fruits for photosynthates which ultimately reduced the fruit yield per plant and fruit yield per plant.
Positive association of yield per plant with average fruit weight, polar diameter and equatorial diameter are in confirmation with findings of Singh(2007) and Prashanth et al., (2008). Positive association of yield per plant with number of locules as also reported by Mahapatra et al., (2013) and pericarp thickness is in accordance with earlier reports of Kumari and Sharma (2013) and Mahapatra et al., (2013). Positive association of yield per plant with fruit volume (Prashanth et $a l ., 2008)$.Equatorial diameter was positively and significantly associated with polar diameter of the fruit (Singh et al., 2008). Pericarp thickness was negatively and significantly associated with plant height at 60 DAT and number of branches at 90 DAT (Fageria and Kohli (1996) and Prashanth et al., (2008) indicating inverse relationship between pericarp thickness and vegetative parameters.

Polar diameter was negatively and significantly associated with plant height 60 DAT (Krishnaprasad and Mathurarai, 1999 and Prashanth et al., 2008), number of branches 90 DAT (Prashanth et al., 2008). Number of locules positively and significantly associated with equatorial diameter (Singh $e t$ al., 1974). It was also positively and significantly associated with plant spread from east to west at $60 \mathrm{DAT}$.

Average fruit weight was inversely associated with plant height at 60 DAT (Fageria and Kohli, 1996), number of branches at 90 DAT (Reddy and Gulshanlal, 1987), plant spread from east to west $90 \mathrm{DAT}$, plant spread from north to south at 90 DAT and plant canopy at 90 DAT. This is attributed to its (average fruit weight) inverse relation with number of fruits, where more competition for photosynthates resulted into reduced fruit size. Fruit volume was positively and significantly associated with polar diameter, equatorial diameter, pericarp thickness and number of locules per 
fruit since all these traits increase the fruit size which in turn increases the fruit volume. But fruit volume was inversely correlated with plant height, number of branches and plant canopy due to increased vegetative growth resulting in decreased fruit size which ultimately reduces fruit volume (Prashanth et al., 2008).

Number of fruits per plant was negatively and significantly associated with polar and equatorial diameter of the fruit, fruit volume and average fruit weight, pericarp thickness, days to first flowering and days to 50 per cent flowering (Sharma et al., 2010) indicates inverse relationship.

Number of seeds per fruit was positively but non significantly associated with plant height at 60 and 90 DAT, number of locules per fruit (Prashanth et al., 2008), days to first flowering and days to 50 per cent flowering. Negative and significant association of plant height and number of branches per plant with polar diameter of the fruit, equatorial diameter of the fruit and average fruit weight could be justified by low mean yield of indeterminate genotypes due to high number of fruits/plant although they possessed smaller fruits and more number of branches/plant. This substained that determinate types were high yielder because of higher average fruit weight they furnished. The correlation coefficient between plant canopy with plant height, number of branches, plant spread from east to west and plant spread from north to south were positively significant at both phenotypic and genotypic level suggesting the interdependence of these traits on each other (Manivannan et al., 2005).

In the present study, path coefficient analysis between the components of yield per plot in tomato was worked out. As the genotypic associations are inherent, the path analysis is discussed only at genotypic level.
In the present investigation, among 21 characters chosen for path analysis number of fruits per plant, average fruit weight, fruit volume, pericarp thickness, equatorial diameter, plant height at 90 DAT, plant spread from north to south at 60 DAT, plant spread from east to west at 60 DAT and days to first flowering had high positive direct effects and positive correlation with total yield. This indicates the true positive association of these traits with total yield. Therefore, direct selection for these traits would reward for improvement of yield.

Number of fruits per plant and average fruit weight had high positive direct effects on total yield (Kumari and Sharma, 2013 and Mahapatra et al., 2013). Number of primary branches per plant and equatorial diameter of the fruit also had high positive direct effects on total yield (Singh and Singh, 2008 and Mahapatra et al., 2013). Plant height (Singh and Singh, 2008) and days to first flowering (Kumari and Sharma, 2013) also had high positive direct effect on total yield. Number of seeds per fruit(Sengupta et al., 2009), yield per plant, polar diameter (Mahapatra et al., 2013), plant canopy at 90 DAT, plant spread from north to south at $60 \mathrm{DAT}$, number of branches 90 DAT, plant canopy at 60 DAT, plant height at 90 DAT and days to 50 per cent flowering(Sharma et al., 2010). had negative direct effects on total yield.

Plant canopy had high negative direct effects as well as negative association with fruit yield indicating that, this character were highly influenced by the environmental factors (Manivannan et al., 2005).Number of branches at 90 DAT was negatively and significantly correlated $\left(\mathrm{r}_{\mathrm{g}}=-0.454\right)$ with total yield and it had negative and high direct effects $(-0.300)$ on total yield, but it had high indirect and negative effects through average fruit weight (-0.699), plant canopy at 60 DAT $(-1.550)$ and plant height at 60 DAT $(-0.544)$ 
and high indirect and positive effects through number of fruits per plant (0.637), Plant height 90 DAT (0.430), Plant spread from north to south 60 DAT (1.041) and Plant spread from east to west 60 DAT (0.580). Under these circumstances, the indirect causal factors also need to be considered simultaneously for selection (Kaushik 2019c).

Table.1 List of genotypes with their codes and sources of collection

\begin{tabular}{|c|c|l|c|c|c|}
\hline Sl. No. & Genotype & Source & Sl. No. & Genotype & Source \\
\hline $\mathbf{1 .}$ & EC 361959 & NBPGR, New Delhi & $\mathbf{1 8 .}$ & EC 630512 & NBPGR, New Delhi \\
\hline $\mathbf{2 .}$ & EC 399667 & NBPGR, New Delhi & $\mathbf{1 9 .}$ & EC 631962 & NBPGR, New Delhi \\
\hline $\mathbf{3 .}$ & EC 570022 & NBPGR, New Delhi & $\mathbf{2 0 .}$ & EC 654725 & NBPGR, New Delhi \\
\hline $\mathbf{4 .}$ & EC 608246 & NBPGR, New Delhi & $\mathbf{2 1 .}$ & EC 654724 & NBPGR, New Delhi \\
\hline $\mathbf{5 .}$ & EC 608250 & NBPGR, New Delhi & $\mathbf{2 2 .}$ & EC 654719 & NBPGR, New Delhi \\
\hline $\mathbf{6 .}$ & EC 608271 & NBPGR, New Delhi & $\mathbf{2 3 .}$ & EC 654699 & NBPGR, New Delhi \\
\hline $\mathbf{7 .}$ & EC 608358 & NBPGR, New Delhi & $\mathbf{2 4 .}$ & EC 638577 & NBPGR, New Delhi \\
\hline $\mathbf{8 .}$ & EC 608362 & NBPGR, New Delhi & $\mathbf{2 5 .}$ & EC 638573 & NBPGR, New Delhi \\
\hline $\mathbf{9 .}$ & EC 608368 & NBPGR, New Delhi & $\mathbf{2 6 .}$ & EC 608288 & NBPGR, New Delhi \\
\hline $\mathbf{1 0 .}$ & EC 608389 & NBPGR, New Delhi & $\mathbf{2 7 .}$ & EC $\mathbf{6 8 6 5 5 4}$ & NBPGR, New Delhi \\
\hline $\mathbf{1 1 .}$ & EC 610652 & NBPGR, New Delhi & $\mathbf{2 8 .}$ & EC 686550 & NBPGR, New Delhi \\
\hline $\mathbf{1 2 .}$ & EC 608465 & NBPGR, New Delhi & $\mathbf{2 9 .}$ & EC 677111 & NBPGR, New Delhi \\
\hline $\mathbf{1 3 .}$ & EC 610654 & NBPGR, New Delhi & $\mathbf{3 0 .}$ & EC 608348 & NBPGR, New Delhi \\
\hline $\mathbf{1 4 .}$ & EC 610655 & NBPGR, New Delhi & $\mathbf{3 1 .}$ & EC 608320 & NBPGR, New Delhi \\
\hline $\mathbf{1 5 .}$ & EC 610661 & NBPGR, New Delhi & $\mathbf{3 2 .}$ & EC $\mathbf{6 8 6 5 4 8}$ & NBPGR, New Delhi \\
\hline $\mathbf{1 6 .}$ & EC 632944 & NBPGR, New Delhi & $\mathbf{3 3 .}$ & EC $\mathbf{6 3 8 5 1 9}$ & NBPGR, New Delhi \\
\hline $\mathbf{1 7 .}$ & EC 634394 & NBPGR, New Delhi & $\mathbf{3 4 .}$ & EC $\mathbf{6 8 6 5 4 4}$ & NBPGR, New Delhi \\
\hline
\end{tabular}

NBPGR - National Bureau of Plant Genetic Resources, New Delhi

Table.1 Contd...

\begin{tabular}{|r|c|c|c|c|c|}
\hline Sl. No. & Genotype & Source & Sl. No. & Genotype & Source \\
\hline $\mathbf{3 5}$ & EC 686543 & NBPGR, New Delhi & $\mathbf{4 8 .}$ & ArkaAbha & IIHR, Bengaluru \\
\hline $\mathbf{3 6 .}$ & EC 677044 & NBPGR, New Delhi & $\mathbf{4 9 .}$ & T-26 & KRCCH, Arabhavi \\
\hline $\mathbf{3 7 .}$ & EC 608269 & NBPGR, New Delhi & $\mathbf{5 0 .}$ & DMT-2 & UAS, Dharwad \\
\hline $\mathbf{3 8 .}$ & EC 686553 & NBPGR, New Delhi & $\mathbf{5 1 .}$ & ARS A-10 & ARS, Arabhavi \\
\hline $\mathbf{3 9}$ & Kashi Hemanth & IIVR, Varanasi & $\mathbf{5 2 .}$ & ARS A-06 & ARS, Arabhavi \\
\hline $\mathbf{4 0 .}$ & Kashi Anupam & IIVR, Varanasi & $\mathbf{5 3 .}$ & ARS A-11 & ARS, Arabhavi \\
\hline $\mathbf{4 1 .}$ & Sel-12 & IARI, New Delhi & $\mathbf{5 4 .}$ & ARS A-05 & ARS, Arabhavi \\
\hline $\mathbf{4 2 .}$ & Hissar Arun & HAU, Hissar & $\mathbf{5 5 .}$ & ARS A-07 & ARS, Arabhavi \\
\hline $\mathbf{4 3 .}$ & Swarna Lalima & HARP, Ranchi & $\mathbf{5 6 .}$ & ARS A-04 & ARS, Arabhavi \\
\hline $\mathbf{4 4 .}$ & Pusa 120 & IARI, New Delhi & $\mathbf{5 7 .}$ & ARS A-09 & ARS, Arabhavi \\
\hline $\mathbf{4 5 .}$ & Pusa Gaurav & IARI, New Delhi & $\mathbf{5 8 .}$ & ARS A-12 & ARS, Arabhavi \\
\hline $\mathbf{4 6 .}$ & HUB 18 & KRCCH, Arabhavi & $\mathbf{5 9 .}$ & ARS A-08 & ARS, Arabhavi \\
\hline $\mathbf{4 7 .}$ & Megha & UAS, Dharwad & $\mathbf{6 0 .}$ & ARS A-13 & ARS, Arabhavi \\
\hline
\end{tabular}

KRCCH - Kittur Rani Channamma College of Horticulture, Arabhavi, IIHR - Indian Institute of Horticulture Research, Bengaluru

ARS - Agricultural Research Station, Arabhavi (Karnataka)

UAS - University of Agricultural Sciences, Dharwad

IIVR - Indian Institute of Vegetable Research, Varanasi
HARP - Horticulture and Agro forestry Research Programme, Ranchi

HAU - Hissar Agricultural University, Hissar 
Table. 2 Genotypic correlation coefficients among growth, earliness, yield and quality parameters in tomato

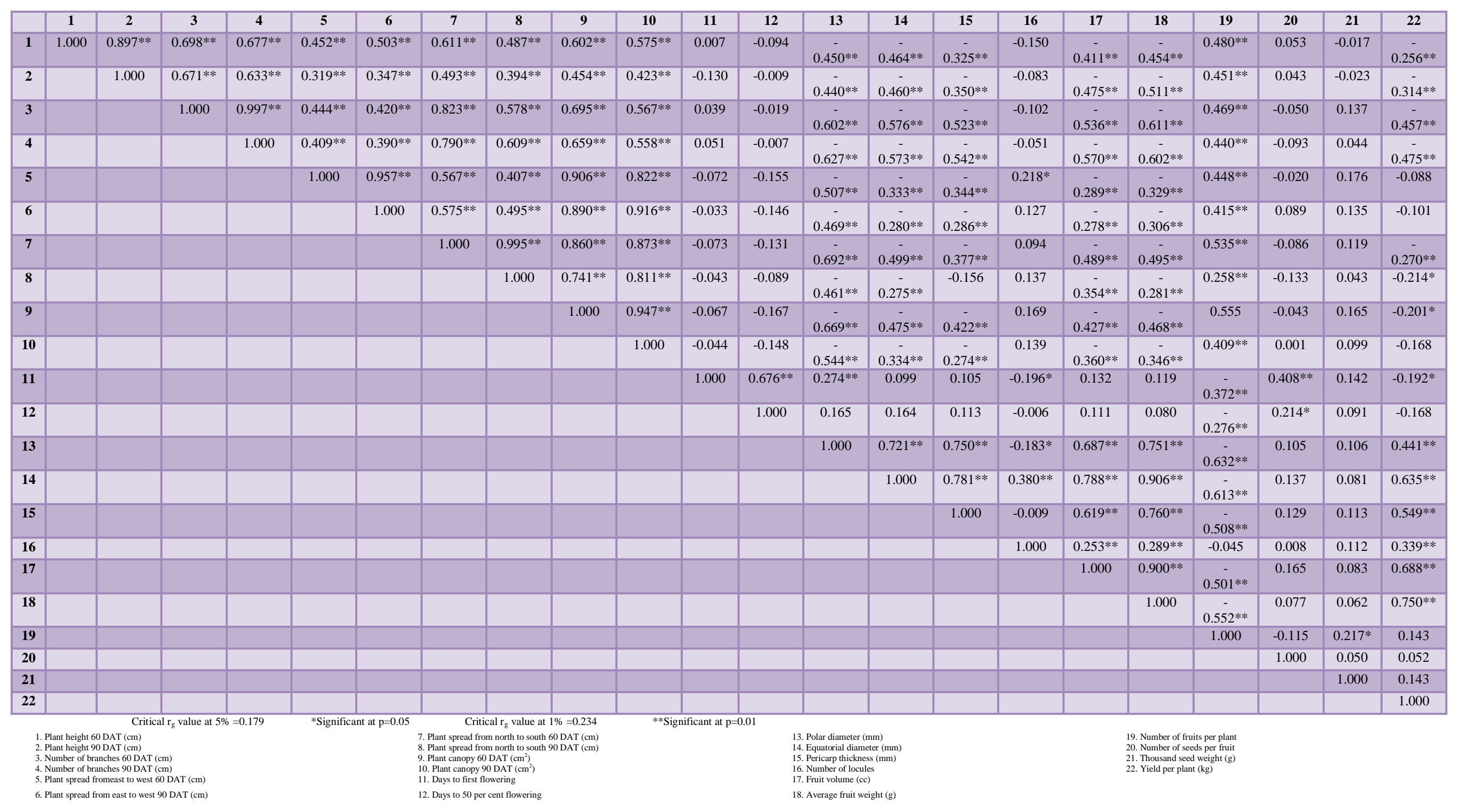




\section{Int.J.Curr.Microbiol.App.Sci (2019) 8(10): 2688-2702}

Table.2a Phenotypic correlation coefficients among growth, earliness, yield and quality parameters in tomato

\begin{tabular}{|c|c|c|c|c|c|c|c|c|c|c|c|c|c|c|c|c|c|c|c|c|c|c|c|c|c|c|c|}
\hline & 1 & 2 & 3 & 4 & 5 & 6 & 7 & 8 & 9 & 10 & 11 & 12 & 13 & 14 & 15 & 16 & 17 & 18 & 19 & 20 & 21 & 22 & 23 & 24 & 25 & 26 & 27 \\
\hline 1 & 1.000 & $\begin{array}{c}0.773 \\
* *\end{array}$ & $\begin{array}{c}0.617 \\
* *\end{array}$ & $\begin{array}{c}0.571 \\
* *\end{array}$ & $\begin{array}{c}0.376 \\
* *\end{array}$ & $\begin{array}{c}0.444 \\
* *\end{array}$ & $\begin{array}{c}0.507 \\
* *\end{array}$ & $\begin{array}{c}0.418 \\
* *\end{array}$ & $\underset{* *}{0.524}$ & $\begin{array}{c}0.527 \\
* *\end{array}$ & 0.007 & $\begin{array}{c}- \\
0.079\end{array}$ & $\begin{array}{c}- \\
0.408 \\
* *\end{array}$ & $\begin{array}{c}- \\
0.422^{*} \\
*\end{array}$ & $\begin{array}{c}- \\
0.29 \\
6 * *\end{array}$ & $\overline{-} .104$ & $\begin{array}{c}- \\
0.384 \\
* *\end{array}$ & $\underset{* *}{-}$ & $\begin{array}{c}0.422 \\
* *\end{array}$ & 0.054 & $\begin{array}{c}- \\
0.019\end{array}$ & 0.168 & $\begin{array}{c}0.269 \\
* *\end{array}$ & 0.001 & $\begin{array}{c}0.195 \\
*\end{array}$ & $\begin{array}{c}- \\
0.243 \\
* *\end{array}$ & $\begin{array}{c}- \\
0.252 \\
* *\end{array}$ \\
\hline 2 & & 1.000 & $\begin{array}{c}0.591 \\
* *\end{array}$ & $\underset{* *}{0.554}$ & $\begin{array}{c}0.281 \\
* *\end{array}$ & $\begin{array}{c}0.293 \\
* *\end{array}$ & $\underset{* *}{0.359}$ & $\begin{array}{c}0.295 \\
* *\end{array}$ & $\begin{array}{c}0.382 \\
* *\end{array}$ & $\begin{array}{c}0.357 \\
* *\end{array}$ & $\begin{array}{c}- \\
0.090\end{array}$ & $\begin{array}{c}- \\
0.018\end{array}$ & $\begin{array}{c}- \\
0.411 \\
* *\end{array}$ & $\begin{array}{c}- \\
0.435^{*} \\
*\end{array}$ & $\begin{array}{c}- \\
0.30 \\
8^{* *}\end{array}$ & $\overline{0 .}$ & $\begin{array}{c}- \\
0.450 \\
* *\end{array}$ & $\begin{array}{c}- \\
0.467 \\
* *\end{array}$ & $\begin{array}{c}0.433 \\
* *\end{array}$ & 0.036 & $\overline{-}-$ & $\begin{array}{c}0.194 \\
*\end{array}$ & $\underset{* *}{0.273}$ & 0.002 & $\begin{array}{c}0.224 \\
*\end{array}$ & $\begin{array}{c}- \\
0.269 \\
* *\end{array}$ & $\begin{array}{c}- \\
0.281 \\
* *\end{array}$ \\
\hline 3 & & & 1.000 & $\begin{array}{c}0.885 \\
* *\end{array}$ & $\begin{array}{c}0.390 \\
* *\end{array}$ & $\begin{array}{c}0.383 \\
* *\end{array}$ & $\underset{* *}{0.613}$ & $\begin{array}{c}0.488 \\
* *\end{array}$ & $\underset{* *}{0.593}$ & $\underset{* *}{0.516}$ & 0.014 & $\begin{array}{c}- \\
0.021\end{array}$ & $\begin{array}{c}- \\
0.558 \\
* *\end{array}$ & $\begin{array}{c}- \\
0.530^{*} \\
*\end{array}$ & $\begin{array}{c}- \\
0.43 \\
8^{* *}\end{array}$ & $\begin{array}{c}- \\
0.073\end{array}$ & $\underset{* *}{-}$ & $\begin{array}{c}-\overline{-563} \\
* *\end{array}$ & $\begin{array}{c}0.432 \\
* *\end{array}$ & $\begin{array}{c}- \\
0.038\end{array}$ & 0.104 & $\begin{array}{c}0.274 \\
* *\end{array}$ & $\begin{array}{c}0.289 \\
* *\end{array}$ & $\begin{array}{c}- \\
0.017\end{array}$ & $\begin{array}{c}0.210 \\
*\end{array}$ & $\begin{array}{c}- \\
0.385 \\
* *\end{array}$ & $\begin{array}{c}- \\
0.402 \\
* *\end{array}$ \\
\hline 4 & & & & 1.000 & $\begin{array}{c}0.346 \\
* *\end{array}$ & $\begin{array}{c}0.337 \\
* *\end{array}$ & $\begin{array}{c}0.626 \\
* *\end{array}$ & $\begin{array}{c}0.488 \\
* *\end{array}$ & $\begin{array}{c}0.570 \\
* *\end{array}$ & $\begin{array}{c}0.490 \\
* *\end{array}$ & 0.062 & $\begin{array}{c}- \\
0.015\end{array}$ & $\begin{array}{c}- \\
0.554 \\
* *\end{array}$ & $\begin{array}{c}- \\
0.521 * \\
*\end{array}$ & $\begin{array}{c}- \\
0.44 \\
6 * *\end{array}$ & -0030 & $\underset{* *}{-}$ & $\begin{array}{c}- \\
0.539 \\
* *\end{array}$ & $\begin{array}{c}0.387 \\
* *\end{array}$ & 0.061 & 0.040 & $\begin{array}{c}0.298 \\
* *\end{array}$ & $\begin{array}{c}0.212 \\
*\end{array}$ & $\begin{array}{c}- \\
0.005\end{array}$ & $\begin{array}{c}0.232 \\
*\end{array}$ & $\begin{array}{c}- \\
0.388 \\
* *\end{array}$ & $\begin{array}{c}- \\
0 . \overline{403} \\
* *\end{array}$ \\
\hline 5 & & & & & 1.000 & $\begin{array}{l}0.811 \\
* *\end{array}$ & $\begin{array}{c}0.448 \\
* *\end{array}$ & $\underset{* *}{0.356}$ & $\begin{array}{c}0.869 \\
* *\end{array}$ & $\begin{array}{c}0.728 \\
* *\end{array}$ & 0.069 & $-\overline{-}$ & $\begin{array}{c}- \\
0.462 \\
* *\end{array}$ & $\begin{array}{c}- \\
0.296 * \\
*\end{array}$ & $\begin{array}{c}- \\
0.32 \\
5^{* *}\end{array}$ & 0.137 & $\begin{array}{c}- \\
0.260 \\
* *\end{array}$ & $\underset{* *}{-}$ & $\begin{array}{c}0.408 \\
* *\end{array}$ & 0.024 & 0.153 & $\begin{array}{c}0.192 \\
*\end{array}$ & 0.098 & 0.086 & 0.097 & $\begin{array}{c}- \\
0.053\end{array}$ & $\begin{array}{c}- \\
0.069\end{array}$ \\
\hline 6 & & & & & & 1.000 & $\begin{array}{c}0.452 \\
* *\end{array}$ & $\begin{array}{c}0.389 \\
* *\end{array}$ & $\begin{array}{c}0.748 \\
* *\end{array}$ & $\begin{array}{c}0.867 \\
* *\end{array}$ & $\begin{array}{c}- \\
0.005\end{array}$ & $\begin{array}{c}- \\
0.088\end{array}$ & $\begin{array}{c}- \\
0.419 \\
* *\end{array}$ & $\begin{array}{c}- \\
0.256^{*} \\
*\end{array}$ & $\begin{array}{c}- \\
0.23 \\
3^{*}\end{array}$ & 0.119 & $\begin{array}{c}- \\
0.238 \\
* *\end{array}$ & $\underset{* *}{0.245}$ & $\begin{array}{c}0.353 \\
* *\end{array}$ & 0.097 & 0.110 & 0.141 & 0.031 & $\begin{array}{c}- \\
0.056\end{array}$ & 0.107 & $\begin{array}{c}- \\
0.046\end{array}$ & $\begin{array}{c}- \\
0.063\end{array}$ \\
\hline 7 & & & & & & & 1.000 & $\begin{array}{c}0.699 \\
* *\end{array}$ & $\begin{array}{c}0.829 \\
* *\end{array}$ & $\begin{array}{c}0.679 \\
* *\end{array}$ & 0.046 & $\stackrel{-}{-} .079$ & $\underset{* *}{-}$ & $\begin{array}{c}- \\
0.401 * \\
*\end{array}$ & $\begin{array}{c}- \\
0.32 \\
3 * *\end{array}$ & 0.097 & $\underset{* *}{-}$ & $\underset{* *}{0.393}$ & $\begin{array}{c}0.396 \\
* *\end{array}$ & 0.062 & 0.048 & $\begin{array}{c}0.207 \\
*\end{array}$ & 0.049 & 0.035 & 0.167 & $\begin{array}{c}- \\
0.218 \\
*\end{array}$ & $\begin{array}{c}- \\
0.236 \\
* *\end{array}$ \\
\hline 8 & & & & & & & & 1.000 & $\begin{array}{c}0.607 \\
* *\end{array}$ & $\begin{array}{c}0.787 \\
* *\end{array}$ & $\begin{array}{c}- \\
0.069\end{array}$ & $\begin{array}{c}- \\
0.061\end{array}$ & $\begin{array}{c}- \\
0.408 \\
* *\end{array}$ & $\begin{array}{c}- \\
0.242 * \\
*\end{array}$ & $\begin{array}{c}- \\
0.13 \\
9\end{array}$ & 0.068 & $\underset{* *}{-}$ & $\begin{array}{c}- \\
0.252 \\
* *\end{array}$ & $\begin{array}{c}0.225 \\
*\end{array}$ & $0 . \overline{-}$ & 0.035 & 0.165 & 0.038 & 0.136 & 0.116 & $\begin{array}{c}- \\
0.179 \\
*\end{array}$ & $\begin{array}{c}- \\
0.204 \\
*\end{array}$ \\
\hline 9 & & & & & & & & & 1.000 & $\begin{array}{c}0.825 \\
* *\end{array}$ & 0.060 & 0.126 & $\begin{array}{c}0.594 \\
* *\end{array}$ & $\begin{array}{c}0.423^{*} \\
*\end{array}$ & $\begin{array}{l}0.39 \\
7 * *\end{array}$ & 0.120 & $\begin{array}{c}0.370 \\
* *\end{array}$ & $\underset{* *}{-}$ & $\underset{* *}{0.481}$ & 0.039 & 0.119 & $\begin{array}{c}0.229 \\
*\end{array}$ & 0.025 & 0.026 & 0.158 & 0.164 & $\begin{array}{c}0.185 \\
*\end{array}$ \\
\hline 10 & & & & & & & & & & 1.000 & $-\overline{-}$ & $\begin{array}{c}- \\
0.102\end{array}$ & $\begin{array}{c}- \\
0.507 \\
* *\end{array}$ & $\begin{array}{c}- \\
0.312 * \\
*\end{array}$ & $\begin{array}{c}- \\
0.24 \\
7 * *\end{array}$ & 0.108 & $\begin{array}{c}- \\
0.330 \\
* *\end{array}$ & $\underset{* *}{-}$ & $\begin{array}{c}0.365 \\
* *\end{array}$ & 0.013 & 0.091 & 0.178 & 0.042 & 0.032 & 0.136 & $\begin{array}{c}- \\
0.137\end{array}$ & $\begin{array}{c}- \\
0.158\end{array}$ \\
\hline 11 & & & & & & & & & & & 1.000 & $\begin{array}{c}0.612 \\
* *\end{array}$ & $\begin{array}{c}0.238 \\
* *\end{array}$ & 0.082 & $\begin{array}{c}0.08 \\
6\end{array}$ & $\begin{array}{c}- \\
0.174\end{array}$ & 0.115 & 0.108 & $\begin{array}{c}- \\
0.328 \\
* *\end{array}$ & $\begin{array}{c}0.385 \\
* *\end{array}$ & $\begin{array}{c}- \\
0.140\end{array}$ & $\begin{array}{c}- \\
0.103\end{array}$ & $\begin{array}{c}- \\
0.122\end{array}$ & 0.023 & 0.064 & $\begin{array}{c}- \\
0.159\end{array}$ & $\begin{array}{c}- \\
0.163\end{array}$ \\
\hline 12 & & & & & & & & & & & & 1.000 & 0.154 & 0.154 & $\begin{array}{c}0.07 \\
8\end{array}$ & $\begin{array}{c}- \\
0.066\end{array}$ & 0.108 & 0.078 & $\begin{array}{c}- \\
0.264 \\
* *\end{array}$ & $\begin{array}{c}0.198 \\
*\end{array}$ & $\begin{array}{c}- \\
0.093\end{array}$ & $\begin{array}{c}- \\
0.277 \\
* *\end{array}$ & 0.161 & $\begin{array}{c}0.199 \\
*\end{array}$ & $\begin{array}{c}- \\
0.042\end{array}$ & $\begin{array}{c}- \\
0.141\end{array}$ & $\begin{array}{c}- \\
0.138\end{array}$ \\
\hline 13 & & & & & & & & & & & & & 1.000 & $\begin{array}{c}0.697 * \\
*\end{array}$ & $\begin{array}{l}0.69 \\
6 * *\end{array}$ & $\overline{0 .}$ & $\begin{array}{c}0.670 \\
* *\end{array}$ & $\begin{array}{c}0.726 \\
* *\end{array}$ & $\begin{array}{c}- \\
0.590 \\
* *\end{array}$ & 0.103 & $\begin{array}{c}- \\
0.085\end{array}$ & $\begin{array}{c}- \\
0.339\end{array}$ & $\begin{array}{c}- \\
0.133\end{array}$ & 0.007 & $\begin{array}{c}- \\
0.161\end{array}$ & $\underset{* *}{0.404}$ & $\begin{array}{c}0.418 \\
* *\end{array}$ \\
\hline 14 & & & & & & & & & & & & & & 1.000 & $\begin{array}{l}0.72 \\
1 * *\end{array}$ & $\begin{array}{c}0.300 \\
* *\end{array}$ & $\begin{array}{c}0.772 \\
* *\end{array}$ & $\begin{array}{c}0.867 \\
* *\end{array}$ & $\begin{array}{c}- \\
0.585\end{array}$ & 0.134 & $\begin{array}{c}- \\
0.076\end{array}$ & $\begin{array}{c}- \\
0.169\end{array}$ & $\begin{array}{c}- \\
0.095\end{array}$ & 0.040 & $0 . \overline{218}$ & $\begin{array}{c}0.545 \\
* *\end{array}$ & $\begin{array}{c}0.570 \\
* *\end{array}$ \\
\hline
\end{tabular}


Table2b Estimates of mean, range, components of variance, heritability and genetic advance for growth and earliness parameters in tomato

\begin{tabular}{|c|c|c|c|c|c|c|c|c|c|c|c|}
\hline Sl. No. & \multicolumn{2}{|c|}{ Character } & $\begin{array}{c}\text { Mean } \pm S \\
\quad \text { Em }\end{array}$ & Range & GV & PV & $\begin{array}{l}\text { GCV } \\
(\%)\end{array}$ & $\begin{array}{l}\text { PCV } \\
(\%)\end{array}$ & $\mathbf{h}^{2}$ & GA & GAM \\
\hline \multicolumn{12}{|c|}{ A. Growth parameters } \\
\hline 1. & \multicolumn{2}{|c|}{ Plant height 60 DAT $(\mathrm{cm})$} & $67.17 \pm 3.18$ & $\begin{array}{l}52.40- \\
116.90\end{array}$ & 136.53 & $\begin{array}{c}156.7 \\
9\end{array}$ & 17.39 & 18.64 & 87.08 & 22.46 & 33.43 \\
\hline 2. & \multicolumn{2}{|c|}{ Plant height 90 DAT (cm) } & $80.84 \pm 2.91$ & $\begin{array}{l}64.07- \\
129.62\end{array}$ & 135.29 & $\begin{array}{c}152.3 \\
0\end{array}$ & 14.38 & 15.26 & 88.82 & 22.58 & 27.93 \\
\hline 3. & \multicolumn{2}{|c|}{ Number of primary branches $60 \mathrm{DAT}$} & $5.34 \pm 0.34$ & $3.10-9.90$ & 1.61 & 1.85 & 23.80 & 25.51 & 87.01 & 2.44 & 45.73 \\
\hline 4. & \multicolumn{2}{|c|}{ Number of primary branches $90 \mathrm{DAT}$} & $8.64 \pm 0.38$ & $6.26-13.05$ & 1.61 & 1.92 & 14.70 & 16.02 & 84.22 & 2.40 & 27.80 \\
\hline 5. & \multicolumn{2}{|c|}{ Plant spread from east to west 60 DAT (cm) } & $50.07 \pm 2.27$ & $36.90-90.80$ & 70.50 & 80.88 & 16.76 & 17.95 & 87.16 & 16.14 & 32.24 \\
\hline 6. & \multicolumn{2}{|c|}{ Plant spread from east to west 90 DAT $(\mathrm{cm})$} & $64.78 \pm 2.41$ & $\begin{array}{l}49.65- \\
102.65\end{array}$ & 88.14 & 99.77 & 14.49 & 15.41 & 88.33 & 18.17 & 28.05 \\
\hline 7. & \multicolumn{2}{|r|}{ Plant spread from north to south 60 DAT $(\mathrm{cm})$} & $49.94 \pm 3.06$ & $39.80-65.50$ & 30.72 & 49.56 & 11.09 & 14.09 & 61.98 & 8.98 & 17.99 \\
\hline 8. & \multicolumn{2}{|r|}{ Plant spread from north to south 90 DAT $(\mathrm{cm})$} & $65.20 \pm 2.49$ & $53.61-85.62$ & 43.06 & 55.51 & 10.06 & 11.42 & 77.57 & 11.90 & 18.26 \\
\hline 9. & \multicolumn{2}{|c|}{ Plant canopy 60 DAT $\left(\mathrm{cm}^{2}\right)$} & $49.84 \pm 2.04$ & $38.43-64.77$ & 36.52 & 44.88 & 12.12 & 13.44 & 81.38 & 11.23 & 22.53 \\
\hline 10. & \multicolumn{2}{|c|}{ Plant canopy 90 DAT $\left(\mathrm{cm}^{2}\right)$} & $64.99 \pm 1.72$ & $54.61-82.37$ & 46.34 & 52.26 & 10.50 & 11.15 & 88.67 & 13.20 & 20.37 \\
\hline \multicolumn{12}{|c|}{ B. Earliness parameters } \\
\hline 1. & \multicolumn{2}{|c|}{ Days to first flowering } & $34.07 \pm 0.61$ & $29.70-39.00$ & 4.04 & 4.78 & 5.90 & 6.42 & 84.42 & 3.80 & 11.16 \\
\hline 2. & \multicolumn{2}{|c|}{ Days to 50 per cent flowering } & $37.99 \pm 0.58$ & $34.00-43.40$ & 4.31 & 5.01 & 5.46 & 5.89 & 86.18 & 3.97 & 10.45 \\
\hline \multicolumn{2}{|c|}{$\begin{array}{l}\text { GV = } \\
\text { Genotypic } \\
\text { variance }\end{array}$} & \multicolumn{5}{|l|}{ GCV = Genotypic coefficient of variance } & \multicolumn{3}{|c|}{$\begin{array}{l}\mathrm{h}^{2}=\text { Heritability } \\
\text { (broad sense) }\end{array}$} & \multicolumn{2}{|c|}{$\begin{array}{l}\text { GAM = Genetic } \\
\text { advance (per cent } \\
\text { mean) }\end{array}$} \\
\hline \multicolumn{2}{|c|}{$\begin{array}{l}\text { PV = } \\
\text { Phenotypic } \\
\text { variance }\end{array}$} & \multicolumn{5}{|l|}{ PCV = Phenotypic coefficient of variance } & \multicolumn{3}{|c|}{$\begin{array}{l}\text { GA = Expected } \\
\text { genetic advance }\end{array}$} & \multicolumn{2}{|c|}{$\begin{array}{l}\text { DAT = Days after } \\
\text { transplanting }\end{array}$} \\
\hline
\end{tabular}


Table.2c Estimates of mean, range, components of variance, heritability and genetic advance for yield parameters in tomato

\begin{tabular}{|c|c|c|c|c|c|c|c|c|c|c|}
\hline $\begin{array}{l}\text { Sl. } \\
\text { No. }\end{array}$ & Character & Mean \pm S. Em & Range & GV & PV & $\begin{array}{l}\text { GCV } \\
(\%)\end{array}$ & $\begin{array}{l}\text { PCV } \\
(\%)\end{array}$ & $\mathbf{h}^{2}$ & GA & GAM \\
\hline \multicolumn{11}{|c|}{ C. Yield parameters } \\
\hline 1. & Polar diameter $(\mathrm{mm})$ & $44.34 \pm 1.42$ & $\begin{array}{l}19.67- \\
59.73\end{array}$ & 91.07 & 95.12 & 21.52 & 21.99 & 95.74 & 19.23 & 43.37 \\
\hline 2. & Equatorial diameter(mm) & $44.21 \pm 1.07$ & $\begin{array}{l}19.00- \\
58.67\end{array}$ & 69.54 & 71.85 & 18.86 & 19.17 & 96.79 & 16.90 & 38.22 \\
\hline 3. & Pericarp thickness(mm) & $4.66 \pm 0.45$ & $2.13-8.10$ & 1.00 & 1.41 & 21.45 & 25.47 & 70.93 & 1.73 & 37.22 \\
\hline 4. & Number of locules & $2.62 \pm 0.28$ & $2.00-4.67$ & 0.37 & 0.53 & 23.28 & 27.80 & 70.10 & 1.05 & 40.16 \\
\hline 5. & Fruit volume (cc) & $35.16 \pm 1.32$ & $2.94-69.21$ & 241.02 & 244.52 & 44.15 & 44.47 & 98.56 & 31.75 & 90.30 \\
\hline 6. & Average fruit weight (g) & $45.87 \pm 3.02$ & $4.44-88.57$ & 356.34 & 374.67 & 41.14 & 42.19 & 95.10 & 37.92 & 82.66 \\
\hline 7. & Number of fruits per plant & $32.67 \pm 2.08$ & $\begin{array}{l}13.86- \\
59.40\end{array}$ & 142.82 & 151.49 & 36.57 & 37.66 & 94.27 & 23.90 & 73.14 \\
\hline 8. & Yield per plant (kg) & $2.04 \pm 0.24$ & $0.35-4.75$ & 0.82 & 0.94 & 44.43 & 47.47 & 87.59 & 1.75 & 85.66 \\
\hline 9. & Yield per plot(kg) & $31.91 \pm 1.28$ & $5.20-68.25$ & 160.31 & 163.61 & 39.67 & 40.08 & 97.97 & 25.81 & 80.89 \\
\hline 10. & Yield per hectare (t) & $39.39 \pm 1.58$ & $\begin{array}{l}6.42- \\
84.25\end{array}$ & 244.34 & 249.38 & 39.67 & 40.08 & 97.97 & 31.87 & 80.89 \\
\hline & GV = Genotypic variance & \multicolumn{2}{|c|}{$\begin{array}{l}\text { GCV = Genotypic coefficient of } \\
\text { variance }\end{array}$} & \multicolumn{3}{|c|}{$\begin{array}{l}\mathrm{h}^{2}=\text { Heritability (broad } \\
\text { sense) }\end{array}$} & \multicolumn{4}{|c|}{$\begin{array}{l}\text { GAM = Genetic advance (per cent } \\
\text { mean) }\end{array}$} \\
\hline & PV = Phenotypic variance & \multicolumn{2}{|c|}{$\begin{array}{l}\mathrm{PCV}=\text { Phenotypic coefficient of } \\
\text { variance }\end{array}$} & \multicolumn{3}{|c|}{$\begin{array}{l}\mathrm{GA}=\text { Expected genetic } \\
\text { advance }\end{array}$} & & & & \\
\hline
\end{tabular}


Table.3 Genotypic path coefficient analysis for total yield per plot in tomato

\begin{tabular}{|c|c|c|c|c|c|c|c|c|c|c|c|c|c|c|c|c|c|c|c|c|c|c|}
\hline & 1 & 2 & 3 & 4 & 5 & 6 & 7 & 8 & 9 & 10 & 11 & 12 & 13 & 14 & 15 & 16 & 17 & 18 & 19 & 20 & 21 & rg \\
\hline 1 & 0.011 & -0.003 & -0.079 & 0.313 & -0.071 & -0.027 & 0.025 & -0.022 & 0.012 & -0.010 & -0.005 & 0.016 & -0.013 & -0.015 & -0.388 & 0.157 & 0.249 & 0.017 & 0.013 & 0.041 & -0.076 & 0.144 \\
\hline 2 & 0.000 & -0.078 & -0.030 & -0.066 & 0.089 & 0.054 & 0.028 & 0.039 & -0.012 & -0.000 & 0.017 & 0.011 & 0.027 & 0.029 & 0.099 & -0.113 & -0.027 & -0.006 & -0.042 & -0.097 & 0.221 & 0.043 \\
\hline 3 & 0.001 & -0.004 & -0.568 & 0.200 & 0.859 & 0.219 & 0.122 & 0.175 & -0.052 & 0.017 & 0.014 & -0.010 & 0.139 & -0.210 & 0.456 & -0.350 & -0.115 & -0.055 & 0.208 & 0.056 & -0.105 & $0.998 * *$ \\
\hline 4 & 0.002 & 0.009 & -0.078 & 1.447 & -0.640 & -0.163 & -0.114 & -0.174 & 0.076 & -0.042 & -0.033 & 0.051 & -0.132 & 0.306 & -1.304 & 0.704 & 0.635 & 0.060 & -0.385 & 0.126 & -0.202 & 0.146 \\
\hline 5 & -0.000 & -0.006 & -0.420 & -0.798 & 1.161 & 0.294 & 0.170 & 0.257 & -0.090 & 0.036 & 0.036 & -0.038 & 0.180 & -0.348 & 1.100 & -0.652 & -0.466 & -0.078 & 0.364 & -0.036 & 0.064 & $0.731 * *$ \\
\hline 6 & -0.000 & -0.013 & -0.381 & -0.724 & 1.045 & 0.326 & 0.138 & 0.224 & -0.082 & 0.037 & 0.046 & -0.034 & 0.171 & -0.323 & 1.004 & -0.644 & -0.410 & -0.069 & 0.330 & -0.050 & 0.071 & $0.663 * *$ \\
\hline 7 & 0.001 & -0.010 & -0.310 & -0.734 & 0.882 & 0.202 & 0.224 & 0.222 & -0.090 & 0.028 & 0.020 & -0.035 & 0.162 & -0.238 & 0.991 & -0.497 & -0.486 & -0.067 & 0.261 & -0.051 & 0.057 & $0.532 * *$ \\
\hline 8 & -0.000 & -0.010 & -0.350 & -0.887 & 1.051 & 0.257 & 0.175 & 0.284 & -0.086 & 0.034 & 0.036 & -0.034 & 0.172 & -0.312 & 1.117 & -0.657 & -0.471 & -0.074 & 0.372 & -0.074 & 0.053 & $0.594 * *$ \\
\hline 9 & -0.001 & -0.008 & -0.246 & -0.913 & 0.872 & 0.224 & 0.168 & 0.205 & -0.120 & 0.056 & 0.060 & -0.058 & 0.188 & -0.299 & 1.573 & -0.911 & -0.718 & -0.077 & 0.361 & -0.075 & 0.149 & $0.429 * *$ \\
\hline 10 & 0.001 & -0.000 & 0.093 & 0.592 & -0.402 & -0.117 & -0.061 & -0.095 & 0.065 & -0.104 & -0.106 & 0.114 & -0.167 & 0.287 & -2.226 & 1.150 & 1.164 & 0.073 & -0.462 & 0.067 & -0.024 & -0.160 \\
\hline 11 & 0.000 & 0.010 & 0.122 & 0.372 & -0.326 & -0.115 & -0.035 & -0.078 & 0.055 & -0.084 & -0.131 & 0.061 & -0.183 & 0.268 & -1.742 & 1.323 & 0.576 & 0.074 & -0.391 & 0.040 & -0.023 & $-0.205^{*}$ \\
\hline 12 & 0.001 & -0.007 & 0.048 & 0.601 & -0.355 & -0.090 & -0.064 & -0.079 & 0.056 & -0.095 & -0.065 & 0.124 & -0.117 & 0.236 & -2.092 & 0.758 & 1.356 & 0.054 & -0.404 & 0.066 & -0.018 & -0.087 \\
\hline 13 & 0.000 & 0.007 & 0.263 & 0.637 & -0.699 & -0.186 & -0.121 & -0.163 & 0.075 & -0.058 & -0.080 & 0.048 & -0.300 & 0.430 & -1.550 & 1.041 & 0.580 & 0.134 & -0.544 & 0.003 & 0.027 & $-0.454 * *$ \\
\hline 14 & -0.000 & -0.003 & 0.175 & 0.652 & -0.593 & -0.155 & -0.078 & -0.130 & 0.053 & -0.044 & -0.051 & 0.043 & -0.190 & 0.680 & -1.066 & 0.650 & 0.451 & 0.086 & -0.721 & 0.004 & -0.070 & $-0.309 * *$ \\
\hline 15 & 0.001 & 0.003 & 0.110 & 0.802 & -0.543 & -0.139 & -0.094 & -0.135 & 0.080 & -0.098 & -0.097 & 0.110 & -0.198 & 0.308 & -2.351 & 1.134 & 1.284 & 0.089 & -0.483 & 0.076 & -0.036 & -0.176 \\
\hline 16 & 0.001 & 0.006 & 0.151 & 0.773 & -0.575 & -0.159 & -0.084 & -0.142 & 0.083 & -0.091 & -0.132 & 0.071 & -0.237 & 0.335 & -2.023 & 1.317 & 0.803 & 0.106 & -0.491 & 0.059 & -0.039 & $-0.266^{* * *}$ \\
\hline 17 & 0.001 & 0.001 & 0.046 & 0.648 & -0.382 & -0.094 & -0.077 & -0.094 & 0.061 & -0.085 & -0.053 & 0.119 & -0.122 & 0.217 & -2.130 & 0.746 & 1.417 & 0.057 & -0.363 & 0.070 & -0.039 & -0.056 \\
\hline 18 & 0.001 & 0.003 & 0.245 & 0.678 & -0.710 & -0.175 & -0.117 & -0.164 & 0.072 & -0.059 & -0.076 & 0.052 & -0.313 & 0.456 & -1.633 & 1.084 & 0.629 & 0.129 & -0.561 & 0.008 & 0.021 & $-0.427 * *$ \\
\hline 19 & -0.000 & -0.004 & 0.147 & 0.694 & -0.527 & -0.134 & -0.073 & -0.132 & 0.054 & -0.060 & -0.064 & 0.062 & -0.203 & 0.611 & -1.414 & 0.805 & 0.640 & 0.090 & -0.803 & 0.043 & 0.004 & $-0.265^{* *}$ \\
\hline 20 & -0.001 & -0.016 & 0.095 & -0.399 & 0.092 & 0.036 & 0.025 & 0.046 & -0.019 & 0.015 & 0.011 & -0.018 & 0.002 & -0.006 & 0.393 & -0.172 & -0.219 & -0.002 & 0.075 & -0.457 & 0.367 & -0.151 \\
\hline 21 & -0.001 & -0.032 & 0.110 & -0.538 & 0.138 & 0.043 & 0.023 & 0.028 & -0.033 & 0.004 & 0.005 & -0.004 & -0.015 & -0.088 & 0.156 & -0.095 & -0.101 & 0.005 & -0.005 & -0.309 & 0.543 & -0.167 \\
\hline & typ & $\begin{array}{l}\text { ation coe } \\
\text { *Signi }\end{array}$ & $\begin{array}{l}\text { icients wi } \\
\text { icant at }\end{array}=$ & $\begin{array}{l}\text { a yield } \mathrm{p} \\
* * \mathrm{~S}\end{array}$ & $\begin{array}{l}\text { t. } \\
\text { ant at }\end{array}$ & $\begin{array}{l}\text { Diagon } \\
.01\end{array}$ & es ind & ect eff & & $=0.049$ & & & & & & & & & & & & \\
\hline & 1 & Thousan & d seed w & $\operatorname{ght}(\mathrm{g})$ & & 7 & icarp thic & ness $(\mathrm{mm}$ & & & & & 13 & Number o & nches & DAT & & & & 19 & th hei & DAT $(\mathrm{cm})$ \\
\hline & 2 & Number & of seeds & er fruit & & 8 & latorial d & meter $(\mathrm{m}$ & & & & & 14 & Plant heig & 0 DAT & & & & & 20 & ys to 50 & cent flowerin \\
\hline & 3 & Yield & er plant & & & 9 & ar diame & $\mathrm{r}(\mathrm{mm})$ & & & & & 15 & Plant canop & $60 \mathrm{DAT}$ & & & & & 21 & ys to first & flowering \\
\hline & 4 & Number & of fruits & er plant & & 10 & nt canol & 90 DAT & $\left.\mathrm{m}^{2}\right)$ & & & & 16 & Plant spre & rom $\mathrm{n}$ & to sout & $60 \mathrm{DA}^{\prime}$ & & & & & \\
\hline & 5 & Average & fruit wei & ht (gm) & & 11 & nt spread & rom nort & to south & 0 DAT & & & 17 & Plant spre: & from ea & to west 6 & DAT $(\mathrm{cl}$ & & & & & \\
\hline & 6 & Fruit $\mathrm{v}$ & olume(cc & & & 12 & nt spread & rom east & o west 9 & $\mathrm{DAT}(\mathrm{cm}$ & & & 18 & Number of & ranche & DAT & & & & & & \\
\hline
\end{tabular}


Plant spread from north to south at 60 DAT was negatively and significantly correlated $\left(r_{g}=-0.266\right)$ with total yield and it had positive and very high direct effects (1.317) on total yield and it had high indirect and negative effects through average fruit weight (-0.575), plant height at 60 DAT (-0.491) and plant canopy at 60 DAT $(-2.023)$ on total yield. Plant height at 90 DAT was negatively and significantly correlated $\left(r_{g}=-0.309\right)$ with total yield and it had positive and high direct effects (0.680) on total yield and it had high indirect and negative effects through average fruit weight (-0.593), plant height at 60 DAT (-0.721) and plant canopy at 60 DAT (-1.066) on total yield. Under these circumstances also, a restricted simultaneous selection model (Singh and Kolkar, 1977) can be followed to nullify the undesirable indirect effects through average fruit weight, plant canopy at 60 DAT and plant height at 60 DAT in order to make use of direct effects of plant height at 90 DAT on total yield.

Polar $\left(r_{g}=0.429\right)$ and equatorial $\left(r_{g}=0.594\right)$ diameter of the fruit were positively and significantly correlated with yield, and these characters had moderate (+) and negligible (-) direct effects on total yield respectively, but both of these traits had high indirect and positive effects through average fruit weight (Prashant et al., 2008), plant canopy at 60 DAT and plant height at 60 DAT. Hence, the indirect causal factors also need to be considered for selection.

Fruit volume, number of fruits per plant, average fruit weight, yield per plot and yield per plant can be improved through direct selection from the existing germplasm, as there is high degree of additive components of variance and high to very high GCV and PCV for these traits.

Yield per plot was positively and significantly associated with yield per plant, polar diameter of the fruit, equatorial diameter of the fruit, number of locules per fruit, pericarp thickness, fruit volume and average fruit weight. Since, these association characters are in desirable direction, selection for these traits may improve the yield per plot. Whereas, yield per plot was negatively and significantly associated with plant height at 60 DAT, plant height at 90 DAT, number of branches at 60 DAT, number of branches at 90 DAT, plant spread from north to south 60 DAT and plant spread from north to south at 90 DAT. It was also negatively and significantly associated with plant spread from east to west at 60 DAT at phenotypic level only.

Correlation study revealed that, yield can be improved by selecting genotypes having more polar and equatorial diameter, number of locules per fruit, pericarp thickness, fruit volume and average fruit weight.

Highest positive direct effects on total yield per plant was shown by number of fruits per plant followed by average fruit weight, fruit volume, pericarp thickness, equatorial diameter, plant height at 90 DAT, plant spread from north to south at 60 DAT, plant spread from east to west at 60 DAT and days to first flowering.

Highest negative direct effects on total yield was shown by plant canopy at 60 DAT followed by plant height at $90 \mathrm{DAT}$, days to 50 per cent flowering, number of seeds per fruit, polar diameter and plant canopy at 90 DAT had negative direct effects on total yield. Characters having high positive direct effects along with positive significant correlation with yield per plant can be directly selected, and simultaneously the characters which show high positive indirect effects can also be selected for the improvement of yield.

\section{References}

Dewey, D.H., and Lu, K.H., 1959, A correlation and path analysis of 
components of crested wheat grass production. Agron. J.,51: 515-518.

Fageria, M.S. and Kohli, U.K. (1996), Correlation studies in tomato - A note. Haryana J. Hort. Sci., 25(3): 158-160.

Kaushik, P., Dhaliwal, S. M., Jindal, K. S., Srivastava, A., Tyagi, V., Brar, S. N., Rana, K. M., 2015. Heterosis and leaf curl virus resistance in rainy season tomato under North Indian conditions. African Journal of Agricultural Research 10: 2763-2772. https://doi.org/10.5897/AJAR2014.9133

Kaushik, P., 2015. Tomato Leaf Curl Virus Resistance in Tomato (Solanum lycopersicum) Hybrids Grown in the Rainy Season under Punjab Conditions. Trends in Biosciences 8(23), Print : ISSN 0974-8431, 6721-6722.

Kaushik, P., Dhaliwal, M., Kaushik, P., Dhaliwal, M.S., 2018. Diallel Analysis for Morphological and Biochemical Traits in Tomato Cultivated under the Influence of Tomato Leaf Curl Virus. Agronomy $\quad 8, \quad 153$. https://doi.org/10.3390/agronomy80801 53

Kaushik, P., 2019. Line $\times$ Tester Analysis for Morphological and Fruit Biochemical Traits in Eggplant (Solanum melongena L.) Using Wild Relatives as Testers. Agronomy $\quad 9, \quad 185$. https://doi.org/10.3390/agronomy90401 85

Kaushik, P., 2019b. Genetic Analysis for Fruit Phenolics Content, Flesh Color, and Browning Related Traits in Eggplant (Solanum melongena). International Journal of Molecular Sciences 20, 2990. https://doi.org/10.3390/ijms20122990

Kaushik, P., 2019c. Application of Conventional, Biotechnological and Genomics Approaches for Eggplant (Solanum melongena.L). Breeding with a Focus on Bioactive Phenolics. https://doi.org/10.4995/Thesis/10251/12 2295

Vijeth, S., Dhaliwal, M. S., Jindal, S. K., Garg, N., Kaushik, P., Sharma, A., 2019. Diallel Analysis of Elite tomato Lines Comprising Leaf Curl Virus Resistance Genes. Applied Ecology and Environmental Research 17(3): 6457-6471.

Krishnaprasad, V.S.R., and Mathurai, 1999, Genetic variation, component association and direct and indirect selections in some exotic tomato germplasm. Indian J. Hort., 59 (3): 262266.

Kumari, S., and Sharma, M.K., 2013, Genetic variability studies in tomato (Solanum lycopersicum L.). Veg. Sci., 40 (1): 8386.

Mahapatra, A.S., Singh, A. K., Vani, V.M., Mishra, R., Kumar, H. and Rajkumar, B.V., 2013, Inter-relationship for various components and path coefficient analysis in tomato (Lycopersicon esculentumMill.). Int. J. Curr. Microbiol. App. Sci.,2 (9): 147-152.

Manivannan, M.I., Prasad, D. and Mir, D.P.M., (2005), Correlation and path coefficient analysis in cherry tomato (Lycopersicon esculentum var. Cerasiforme). New Agriculturist, 16 (2): 151-154.

Prashanth, S.J., Jaiprakashnarayan, R.P., Mulge, R. and Madalageri, M,B., 2008, Correlation and path analysis in tomato (Lycopersicon esculentum Mill.). Asian J. Hort., 3 (2): 403-408.

Reddy, M.L.N. and Gulshanal, 1987, Genetic variability and path coefficient analysis in tomato (Lycopersicon esculentum Mill.) under summer season. Prog. Hort., 19 (3-4): 284-288.

Sengupta, S.K., Mehta, A. K. and Naidu, A.K., 2009, Genetic studies for fruit yield and its components in tomato 
(Lycopersicon esculentum Mill.). Haryana J. Hort. Sci., 38 (1-2): 112114.

Sharma, J.P., Singh, A.K. and Tiwari, S.P., 2010, Selection parameters for productive plant type in tomato (Lycopersicon esculentum Mill.). J. Hill Agric., 1 (1): 52-55.

Singh, A.K., 2007, Correlation and path coefficient studies in tomato under cold arid conditions of ladakh. Haryana J. Hort. Sci., 36 (3-4): 346-347.

Singh, A.K., 2009, Genetic variability, heritability and genetic advance studies in tomato under cold arid region of Ladakh. Indian J. Hort., 66 (3): 400403.

\section{How to cite this article:}

Sunilkumar, M.K., S. Vijeth, Vijayakumar Rathod and Prashant Kaushik. 2019. Genetic Associations Analysis in Tomato (Solanum lycopersicum L.) Involving Improved Germplasm Lines for Agronomic and Yield Contributing Traits. Int.J.Curr.Microbiol.App.Sci. 8(10): 26882702. doi: https://doi.org/10.20546/ijcmas.2019.810.310 